\title{
Hair Follicle Specific ACVR1/ALK2 Critically Affects Skin Morphogenesis and Attenuates Wound Healing
}

\author{
(1)) \\ Michael Sorkin ${ }^{1}$, Shailesh Agarwal ${ }^{1}$, Kavitha Ranganathan ${ }^{1}$, Shawn Loder ${ }^{1}$, David Cholok ${ }^{1}$, \\ David Fireman ${ }^{1}$, John Li, ${ }^{1}$ Shuli Li ${ }^{1}$, Bin Zhao ${ }^{3}$, Yuji Mishina ${ }^{2}$, Paul Cederna ${ }^{1}$, Benjamin Levi ${ }^{1,4}$

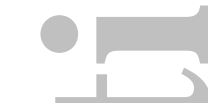 \\ ${ }^{1}$ Division of Plastic and Reconstructive Surgery, Department of Surgery, University of Michigan, Ann Arbor, MI \\ ${ }^{2}$ Department of Biologic and Material Sciences, School of Dentistry, University of Michigan, Ann Arbor, MI \\ ${ }^{3}$ Albert Einstein College of Medicine, Department of Genetics, Bronx, NY \\ $<$

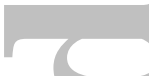 \\ ${ }^{4}$ Corresponding Author: \\ Benjamin Levi, MD \\ Department of Surgery \\ University of Michigan Health System \\ 1500 East Medical Center Drive \\ 2130 Taubman Center \\ SPC 5340 \\ Ann Arbor, MI 48109-0219 \\ E-mail:blevi@umich.edu \\ B.L. received funding from NIH/NIGMS -K08GM109105-0, Plastic Surgery Foundation \\ National Endowment Award, the Association for Academic Surgery Roslyn Award American \\ Association for the Surgery of Trauma Research \& Education Foundation Scholarship, Plastic \\ Surgery Foundation, American College of Surgeons. A.S and K.R. received NIF F32 Fellowship \\ grants.
}

This is the author manuscript accepted for publication and has undergone full peer review but has not been through the copyediting, typesetting, pagination and proofreading process, which may lead to differences between this version and the Version record. Please cite this article as doi:10.1111/ wrr.12549. 


\section{Abstract}

The bone morphogenic protein signaling (BMP) is intricately involved in the quiescence and regulation of stem cells through activation of BMP receptors. Hair follicle stem cells play a critical role in cutaneous homeostasis and regeneration. Here, we utilize a novel mouse model with targeted overexpression of the BMP receptor ALK2/ACVR1 in hair follicle stem cells, to characterize its role in skin development and postnatal wound healing. Initial histologic evaluation demonstrated significant dysregulation in hair follicle morphogenesis in mutant mice. These demonstrated increased numbers of individual hair follicles with altered morphology and localization. Mutant follicles were found to exhibit elevated proliferative activity as well as increased prevalence of CD34 and ITGA6 positive follicle stem cells. Interestingly, constitutive overexpression of ALK2 resulted in attenuation of cutaneous wound healing. These findings demonstrate that hair follicle specific ALK2 is intricately involved in maintenance of the stem cell niche and wound healing.

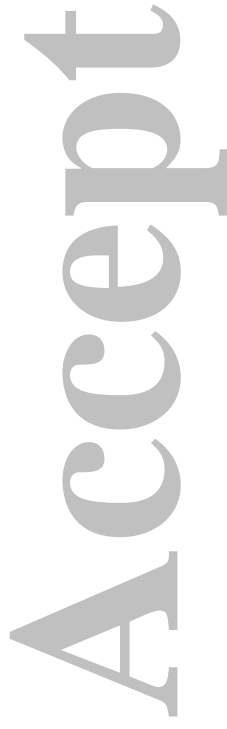




\section{Introduction}

Human skin is composed of an intricate microenvironment that provides niches to several adult stem cell populations (1). Hair follicles represent a unique source of stem cells that are maintained through self-renewal and differentiation during the hair cycle (2). In this process, hair follicle morphogenesis proceeds through a sequence of proliferation (anagen), destruction (catagen) and stasis (telogen) $(3,4)$. The hair cycle itself is predominantly regulated by cells, which reside within the hair bulge, a morphologically distinct compartment of the mature hair follicle. These bulge stem cells are marked by expression of CD34, Itga6, Lhx2, Tcf3, and Sox9 (5).

NFATc1, a calcium sensitive transcription factor, has previously been implicated in the maintenance of hair bulge stem cells that are predominantly quiescent. In fact, inhibition of NFATc1 through cyclosporine A as well as genetic deletion causes premature cell cycle activation and precocious entry into the anagen phase $(6,7)$. While the exact mechanisms driving hair stem cell regulation and quiescence are still unknown, multiple signaling pathways have been implicated to play an important role in this process. Kandyba et al have shown that Smad 1 and Smad 5, both key effectors of the canonical BMP pathway, are required for undisturbed follicle morphogenesis, and that deletion of Smad1/5 or the BMPR1a receptor results in dysfunctional hair follicle morphogenesis as well as postnatal hair shaft differentiation (8). These factors can also affect wound healing (9).

To more clearly define the role of BMP on hair follicle stem cell morphology, cycle regulation, and wound healing, we utilized a transgenic mouse model that is characterized by constitutive expression of the BMP receptor ALK2/ACVR1 under the conditional control of the hair bulge cell specific promoter NFATc1. A better understanding of these processes may 
provide the basis for developing therapeutic approaches that modulate BMP signaling to promote wound healing.
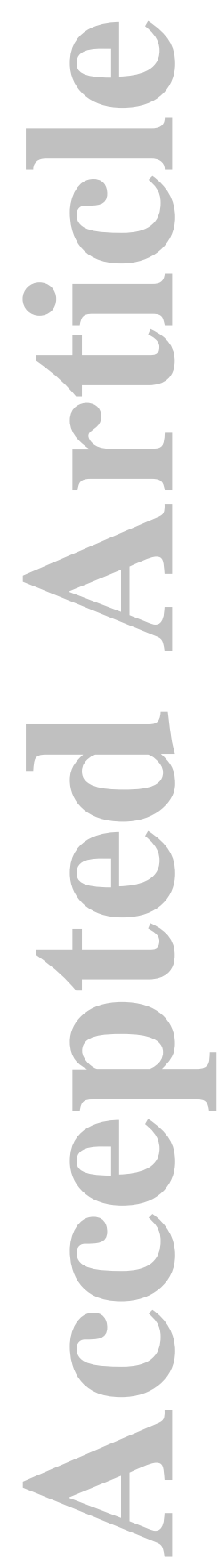


\section{Methods}

Animals

We have previously developed a transgenic mouse model that carries a mutation resulting in overexpression of ALK2 in the setting of Cre recombinase expression driven by the NFATc1 promoter (10). Eight-week old female mice were used for all aspects of this study with nonmutant littermate controls that did not carry the NFATc1 cre transgene. All animal procedures were performed in accordance with the guidelines in the Guide for the Use and Care of Laboratory Animals from the Institute for Laboratory Animal Research (ILAR, 2011) and were approved by the Institutional Animal Care and use Committee of the University of Michigan (PRO0001553).

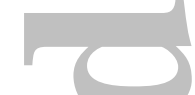

Skin Injury

Mice were anesthetized according to protocol using inhaled isofluorane. Dorsal hair was removed, and two $6 \mathrm{~mm}$ full thickness circular wounds were created equidistant from the midline. Round shaped silicone rings were applied to the wounds and secured with 6-0 Nylon suture to prevent skin contraction from the panniculus carnosus. Wounds were then covered with an occlusive Tegaderm dressing (3M, Maplewood, MN). caALK2 NFATc1 mutant mice $(\mathrm{n}=8)$ and littermate control mice $(\mathrm{n}=8)$ underwent the same treatment. Buprenorphine $(0.01 \mathrm{mg} / \mathrm{kg}$, Buprenex; Reckitt Benckiser Pharmaceuticals Inc, Richmond, VA) was administered by subcutaneous injection every 12 hours for the first 72 hours after injury. Wound healing was evaluated every other day obtaining standardized photographs. For this purpose, the occlusive dressing was removed each time and then replaced. Wound healing curves were generated using photometric analysis with ImageJ software (NIH, Bethesda, MD). 
Histology

Dorsal skin from mutant and littermate control mice was harvested, fixed in formalin for 24 hours, and embedded in paraffin prior to sectioning. Histology was performed as previously described, including H\&E and immunohistochemistry for pSMAD and Ki67 (Santa Cruz, CA). Images were taken with a Nikon E-800 upright microscope (Nikon, Melville, NY) utilizing 10x and 40x bright field microscopy.

Flow cytometry

Dorsal skin was harvested from mutant and littermate control mice ( $\mathrm{n}=3$ per group) as previously described. The tissues were dissected, finely minced, and enzymatically digested in a $0.075 \%$

type 1 collagenase solution (Sigma-Aldrich, St. Louis, MO) and the stained using CD34

(eBioscience) and Itga6 (eBioscience) antibodies. Flow cytometry was performed on a BD

FACS Aria (BD Biosciences) and subsequently analyzed using FloJo software (Tristar, Ashland, OR).

Statistical analysis

Statistical significance was determined using the student's unpaired $t$-test. A $p$-value $<0.05$ was considered statistically significant. 


\section{Results}

\section{Constitutive expression of caALK2 affects hair follicle morphology.}

Mutant mice overexpressing ALK2 within NFATc1 positive cells demonstrated distinct phenotypic differences when compared to littermate controls. Their hair was noted to be lighter in color and more disoriented in alignment. Furthermore, mutant mice did not fully lose their hair when treated with depilatory cream indicating deeper-rooted hair follicles (Figure 1a). Based on H\&E staining, we found that the number of hair follicles observed in mutant skin was significantly increased (Figure 1c) and, the hair follicles appeared highly dysmorphic with large cell rich clusters localizing in the subcuticular fat just superficial to the panniculus carnosus (Figure 1d). The localization of hair follicles in this layer is commonly observed during the proliferative anagen phase of the hair cycle and may indicate that the mutant hair follicles are arrested in this phase and cannot transition further. In order to identify NFATc1 expression in the skin, we utilized a double fluorescent reporter mouse model that was cross-bred with NFATc1cre mice. In the resulting mice, all cells that express the NFTAc1 gene were labeled green, while all other cells are red. Interestingly, we were able to directly localize the NFATc1 expressing cells within the hair follicle and specifically the hair bulb (Figure 1e).

\section{Over-activation of the BMP pathway results in aberrant regulation of cell proliferation, stem cell maintenance and wound healing}

Immunofluorescence performed on the skin demonstrated increased pSMAD expression in the mutant mice throughout the hair follicle and within the hair bulb located in the deeper subcuticular layer as compared to the controls (Figure 2a). 
After isolating cells from the skin of mutant and control mice using flow cytometry, we evaluated the expression of Integrin 6 and CD34 within these cell populations, since these markers have been shown to represent hair follicle stem cells. The number of cells as identified by these markers was increased in the mutant group, although not statistically significant (Figure 2c). Furthermore, expression of proliferation marker Ki67 was also localized to the hair bulb region of the anagen hair follicles in the mutant mice and co-localized with GFP positive NFATc1 cells (Figure 2b). To assess the effect of caALK2 constitutive expression on wound healing, we utilized a well-established mouse model that prevents healing by primary contraction. We observed a significant attenuation of the wound healing process in the mutant mice compared to the controls, which resulted in delayed wound healing (Figure 2d).

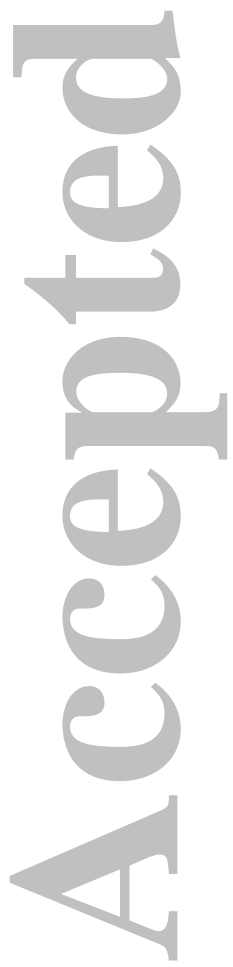




\section{Discussion}

A balance of numerous signaling pathways determines the architecture and organization of the hair follicle. In this study, we demonstrate that ALK2 overexpression within NFATc1 cells of the hair follicle resulted in an increased number and size of individual follicles with an aberrant morphology and function. Our results are consistent with those of previous studies that highlight the importance of homeostasis within the hair cycle and tissue regeneration. Rishikaysh et al. demonstrate that BMP and Wnt signaling is important for maintaining the barrier function of skin in that an imbalance of these factors results in a prolonged anagen phase within the hair follicle (11). A disturbance in homeostasis within these pathways results in loss of stem cell function and hair loss in mice (12). The adult hair follicle consists of an upper permanent segment, and a lower basal layer that undergoes intermittent growth and regression with each cycle. The anagen phase requires local activation of hair follicle cells within the bulge of the hair follicle, and BMP promotes quiescence of the stem cells within the hair follicle niche $(8,13)$. In our model specifically, stabilization of Alk2 resulted in quiescence of the local hair follicle stem cells located within the bulge. While the number and size of each individual hair follicle was increased in our mutant model, this does not directly correlate with function. In fact, the regenerative function and ability to transition through the hair cycle is likely inhibited as a result of overexpression of Alk2. The mechanism by which this occurs is unclear to date, but likely involves a direct interaction between Alk2 and the bulge stem cells specifically. Peters et. al showed a similar indirect relationship between hair follicle number and function in the setting of ceramide synthase 4 inhibition; loss of ceramide synthase 4 , a factor that is also affected by BMP and Wnt signaling, resulted in inappropriate activation and subsequent exhaustion of human hair follicle stem cells with enlargement of the sebaceous glands (12). Gnedeva et al. 
showed that BMP signaling in a homeostatic fashion is critical for hair growth, and that use of the BMP inhibitor dorsomorphin completely eliminated hair-inducing activity from cells of dermal papilla origin (14). Thus, an important balance is required for proper hair cycle maintenance. Furthermore, modulation of the hair follicle cycle is affected by the local niche and cell populations within the hair follicle itself. Under the influence of stabilized Alk2, the expression of Integrin 6 and CD34 was increased in the mutant mice compared to the control mice. Such differences may explain why the mutant stem cells have altered function in terms of hair follicle cycle maintenance and tissue regeneration in comparison to the control mice (6). While our findings suggest the importance of the BMP pathway on gross wound healing, a more in depth histologic and biomolecular analysis of the phases of wound healing is required in order to understand the underlying mechanism. Further studies will therefore be needed to establish a link between hair follicle morphogenesis, function, and tissue regeneration.

While it is apparent that function of hair follicle stem cells is affected by the overexpression of Alk2, additional studies are required to more clearly delineate a direct relationship between these variables. By understanding the role of Alk2 on local stem cell populations within the hair follicle niche, we can harness these findings to optimize tissue regeneration. 
Figure Legends:

Figure 1: NFATc1 regulated caALK2 overexpression results in aberrant hair morphology. (A) Photographs of NFATc1/caALK2 mutant mice and litter mate controls after depilation. (B) HE staining of adult skin with quantification of (C) hair follicle number and (D) hair follicle size. (E) Immunofluorescence of skin and hair follicle in double transgenic reporter mouse NFATc1Cre/ROSA26mTmG. Values are expressed as mean \pm SEM. * $\mathrm{p}<0.05$.

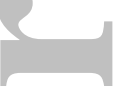

Figure 2: Hair follicle ALK2 critically regulates cell proliferation, stem cell maintanence and wound healing. (A) Immunofluorescence for pSmad 1/5/8 in adult mutant and wild-type skin. (B) Immunofluorescence for proliferation marker Ki67. (C) Flow cytometry quantification of cells expressing CD34 and Itga6. (D) Wound healing curve and corresponding representative photographs demonstrate attenuated wound closure in mutant mice $(n=8)$. Values are expressed as mean \pm SEM. * $\mathrm{p}<0.05$.

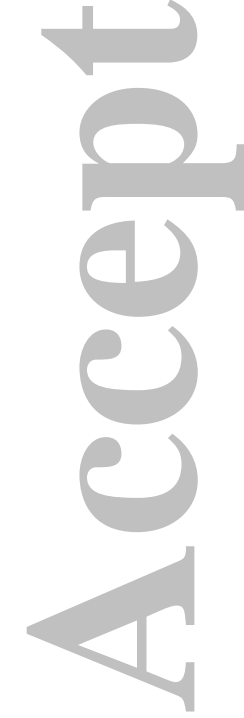


References:

1. Gurtner GC, Werner S, Barrandon Y, Longaker MT. Wound repair and regeneration. Nature. 2008;453(7193):314-21.

2. Alonso L, Fuchs E. The hair cycle. J Cell Sci. 2006;119(Pt 3):391-3.

3. Muller-Rover S, Handjiski B, van der Veen C, Eichmuller S, Foitzik K, McKay IA, et al. A comprehensive guide for the accurate classification of murine hair follicles in distinct hair cycle stages. J Invest Dermatol. 2001;117(1):3-15.

4. Hsu YC, Li L, Fuchs E. Emerging interactions between skin stem cells and their niches. Nat Med. 2014;20(8):847-56.

5. Mardaryev AN, Meier N, Poterlowicz K, Sharov AA, Sharova TY, Ahmed MI, et al. Lhx2 differentially regulates Sox9, Tcf4 and Lgr5 in hair follicle stem cells to promote epidermal regeneration after injury. Development. 2011;138(22):4843-52.

6. Keyes BE, Segal JP, Heller E, Lien WH, Chang CY, Guo X, et al. Nfatc1 orchestrates aging in hair follicle stem cells. Proc Natl Acad Sci U S A. 2013;110(51):E4950-9.

7. Horsley V, Aliprantis AO, Polak L, Glimcher LH, Fuchs E. NFATc1 balances quiescence and proliferation of skin stem cells. Cell. 2008;132(2):299-310.

8. Kobielak K, Stokes N, de la Cruz J, Polak L, Fuchs E. Loss of a quiescent niche but not follicle stem cells in the absence of bone morphogenetic protein signaling. Proc Natl Acad Sci U S A. 2007;104(24):10063-8.

9. Lewis CJ, Mardaryev AN, Poterlowicz K, Sharova TY, Aziz A, Sharpe DT, et al. Bone morphogenetic protein signaling suppresses wound-induced skin repair by inhibiting keratinocyte proliferation and migration. J Invest Dermatol. 2014;134(3):827-37. 
10. Agarwal S, Loder SJ, Brownley C, Eboda O, Peterson JR, Hayano S, et al. BMP signaling mediated by constitutively active Activin type 1 receptor (ACVR1) results in ectopic bone formation localized to distal extremity joints. Dev Biol. 2015;400(2):202-9.

11. Rishikaysh P, Dev K, Diaz D, Qureshi WM, Filip S, Mokry J. Signaling involved in hair follicle morphogenesis and development. Int J Mol Sci. 2014;15(1):1647-70.

12. Peters F, Vorhagen S, Brodesser S, Jakobshagen K, Bruning JC, Niessen CM, et al. Ceramide synthase 4 regulates stem cell homeostasis and hair follicle cycling. J Invest Dermatol. 2015;135(6):1501-9.

13. Genander M, Cook PJ, Ramskold D, Keyes BE, Mertz AF, Sandberg R, et al. BMP signaling and its pSMAD1/5 target genes differentially regulate hair follicle stem cell lineages. Cell Stem Cell. 2014;15(5):619-33.

14. Gnedeva K, Vorotelyak E, Cimadamore F, Cattarossi G, Giusto E, Terskikh VV, et al. Derivation of hair-inducing cell from human pluripotent stem cells. PLoS One. 2015;10(1):e0116892. 
A
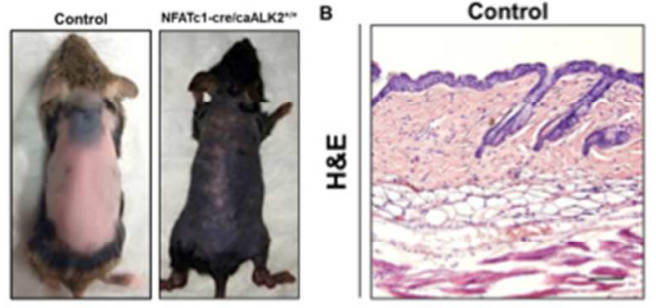

NFATc1-cre/caALK2 ${ }^{*+*}$

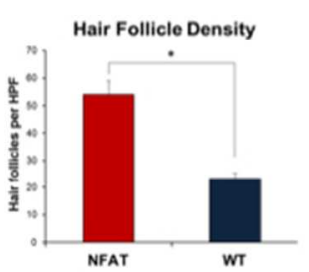

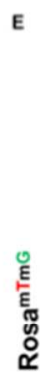
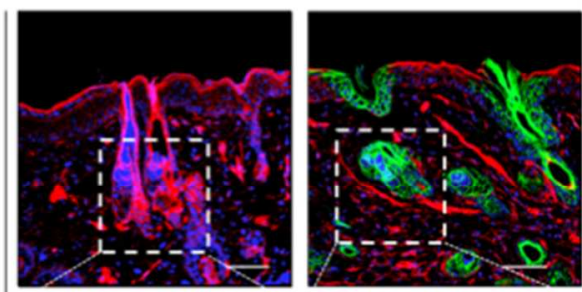

D

Hair Follicle Size
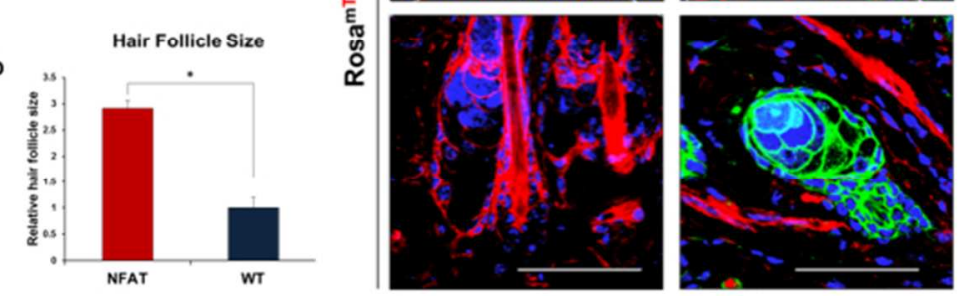

$60 \times 45 \mathrm{~mm}(300 \times 300 \mathrm{DPI})$

Wound Repair and Regeneration

This article is protected by copyright. All rights reserved. 

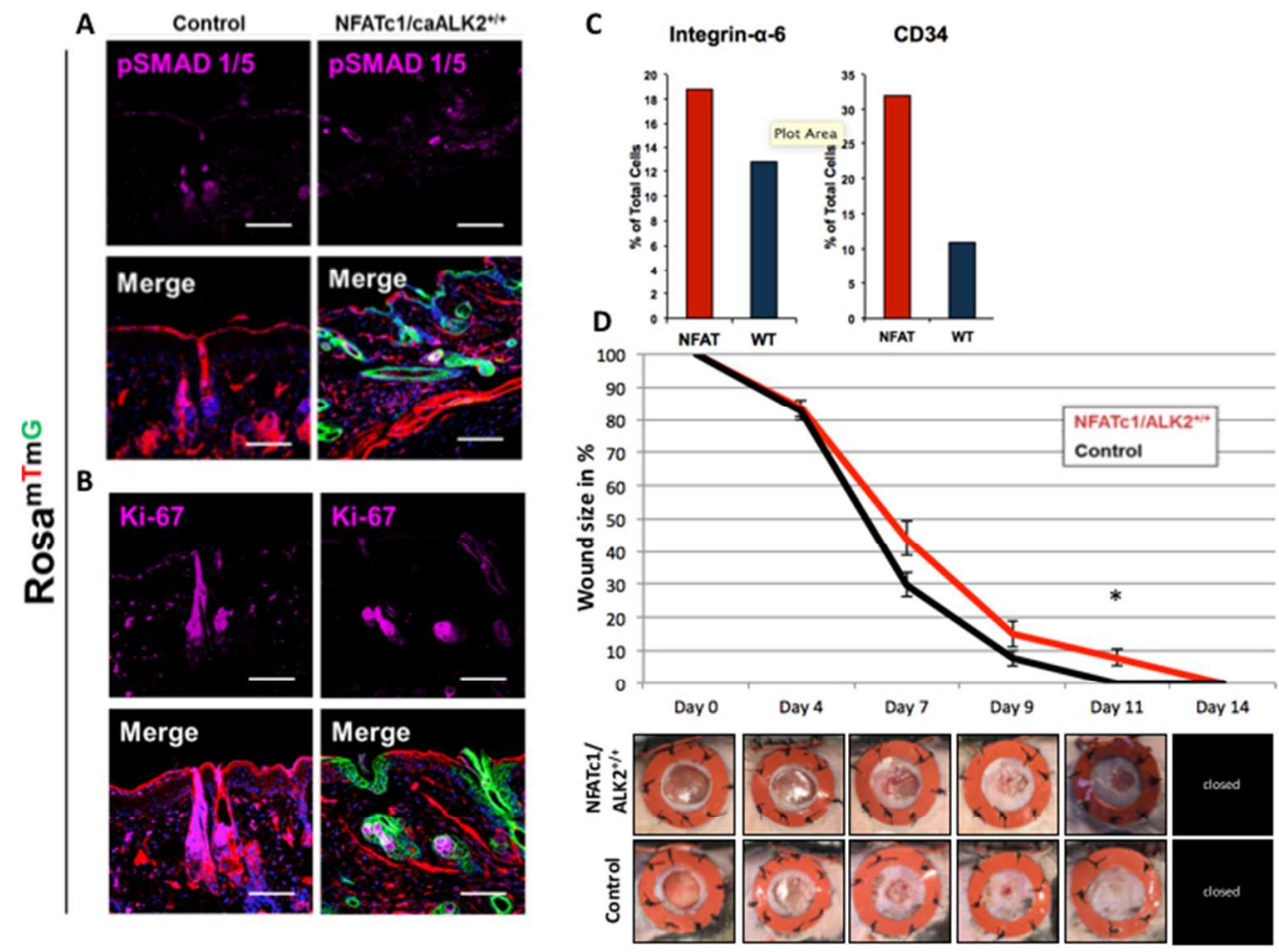

$60 \times 45 \mathrm{~mm}(300 \times 300$ DPI $)$ 